\section{Bleeding jejunal lymphangioma diagnosed by double-balloon enteroscopy}

Obscure gastrointestinal bleeding poses a diagnostic challenge to the physician. When examination of the colon and the upper gastrointestinal tract reveals no definite bleeding source, the small bowel becomes the focus of investigation. The small bowel is difficult to investigate endoscopically because of its extraordinary length, but the recent advent of doubleballoon enteroscopy has made it possible to examine this section of the gastrointestinal tract thoroughly, which should increase the diagnostic yield [1-5]. We present here the first reported case of a jejunal lymphangioma diagnosed by double-balloon enteroscopy.

A 75-year-old woman was admitted for the management of obscure gastrointestinal bleeding and microcytic anemia. She had a history of tarry stools and exercise intolerance for 2 years. Esophagogastroduodenoscopic and colonoscopic examinations had been performed elsewhere and had revealed no definite bleeding source. One month before admission to our unit, repeat esophagogastroduodenoscopy in our hospital revealed reflux esophagitis and atrophic gastritis and repeat colonoscopy revealed small diverticula in the ascending and transverse colon without any evidence of active bleeding.

After admission, a technetium-labeled red blood cell scan suggested possible active bleeding in the jejunum. Push-type enteroscopy was performed to the level of $90 \mathrm{~cm}$ beyond the ligament of Treitz but did not reveal a bleeding source. Small-bowel barium radiography and computed tomographic scans of the abdomen and pelvis were negative. The patient underwent double-balloon enteroscopy (Fujinon EN-450P5/20; Fujinon Corp., Saitama, Japan) under midazolam sedation. This examination revealed a circumferential lesion extending over a 4-cm-long segment in the distal jejunum with yellowish thickened mucosal folds in a cobblestone configuration, covered with fresh blood clot (Figure 1). Histological examination of a biopsy specimen

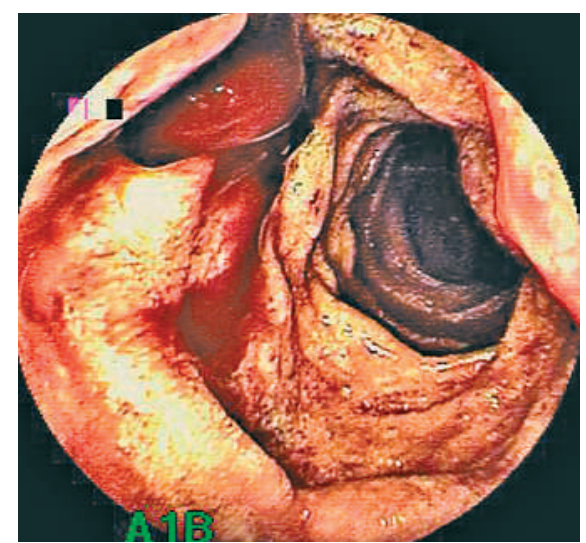

Figure 1 Enteroscopic view of the jejunal tumor: a circumferential lesion with thickened mucosal folds and coated with blood clot was observed in a segment of the distal jejunum.

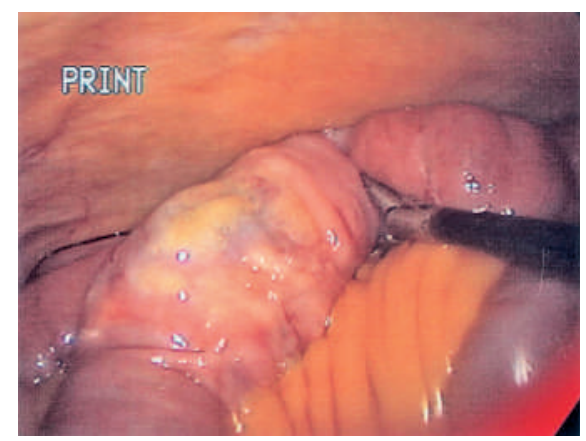

Figure 2 Laparoscopic view of the jejunal tumor: a 4-cm segment of jejunum with an uneven serosal surface was noted $200 \mathrm{~cm}$ proximal to the ileocecal junction.

showed dilated lymphatic channels in the lamina propria.

For further management of chronic bleeding, she underwent a laparoscopy-assisted operation, when a submucosal tumor measuring $6 \mathrm{~cm} \times 4 \mathrm{~cm}$ was found $200 \mathrm{~cm}$ proximal to the ileocecal junction (Figure 2,3). Histopathological examination of the tumor revealed a lymphangioma involving both the mucosa and the submucosa (Figure 4). The patient made an uneventful recovery and was discharged 1 week after surgery. She has been symptom-free since then.

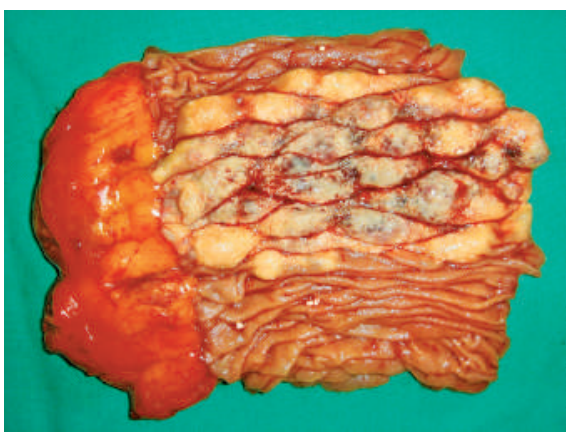

Figure 3 The mucosal aspect of the jejunal tumor. There was circumferential thickening of the mucosal folds, with a cobblestone appearance and a variegated color pattern.

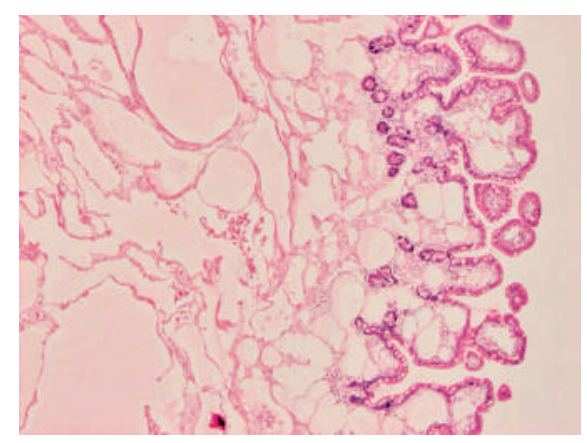

Figure 4 Histopathological view of the tumor. Dilated lymphatic channels were noted within the mucosa and submucosa, with some lymphocytes mixed with the lymph in the spaces (hematoxylin and eosin stain, $\times 20$ magnification).

\section{S.-J. Hsu', Y.-T. Chang', M.-C. Chang', S.-C. Yü ${ }^{2}$ J.-C. Lee ${ }^{3}$, C.-T. Shun ${ }^{3}$,}

\section{J.-M. Wong ${ }^{1}$}

${ }^{1}$ Department of Internal Medicine, National Taiwan University Hospital and College of Medicine, National Taiwan University, Taipei, Taiwan

${ }^{2}$ Department of Surgery, National Taiwan University Hospital and College of Medicine, National Taiwan University, Taipei, Taiwan

${ }^{3}$ Department of Pathology, National Taiwan University Hospital and College of Medicine, National Taiwan University, Taipei, Taiwan. 


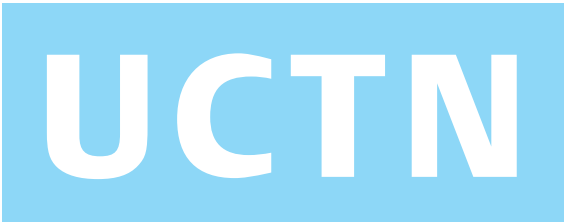

\section{References}

${ }^{1}$ Yamamoto H, Sekine Y, Sato Y et al. Total enteroscopy with a nonsurgical steerable double-balloon method. Gastrointest Endosc 2001; 53: 216-220

2 Yamamoto H, Kita H, Sunada K et al. Clinical outcomes of double-balloon endoscopy for the diagnosis and treatment of small-intestinal diseases. Clin Gastroenterol Hepatol 2004; 2: 1010-1016

${ }^{3}$ Hadithi M, Heine GD, Jacobs MA et al. A prospective study comparing video capsule endoscopy with double-balloon enteroscopy in patients with obscure gastrointestinal bleeding. Am J Gastroenterol 2006; 101: $52-57$
${ }^{4}$ Heine GD, Hadithi M, Groenen MJ et al. Double-balloon enteroscopy: indications, diagnostic yield, and complications in a series of 275 patients with suspected small-bowel disease. Endoscopy 2006; 38: 42 - 48

${ }^{5}$ Nakamura M, Niwa Y, Ohmiya $\mathrm{N}$ et al. Preliminary comparison of capsule endoscopy and double-balloon enteroscopy in patients with suspected small-bowel bleeding. Endoscopy 2006; 38: 59-66
Corresponding author

\section{J.-M. Wong, M.D.}

Department of Internal Medicine National Taiwan University Hospital 7 Chung Shan South Road

Taipei

Taiwan

Fax: $\quad+886-2-23947927$

Email: jmwong@ha.mc.ntu.edu.tw 